\title{
Endodermal cyst: rare intraaxial brainstem location. Illustrative cases
}

\author{
Atul Goel, MCh, ${ }^{1}$ Dikpal Jadhav, MCh, ${ }^{1}$ Abhidha Shah, MCh, ${ }^{1}$ Asha Shenoy, MD, ${ }^{2}$ and Naina Goel, MD ${ }^{2}$ \\ Departments of ${ }^{1}$ Neurosurgery and ${ }^{2}$ Neuropathology, K.E.M. Hospital and Seth G.S. Medical College, Parel, Mumbai
}

BACKGROUND This report detailed an extremely rare location of an endodermal cyst. Such location of the tumor can pose radiological confusion and a therapeutic dilemma. However, when identified, it can be a pleasant surprise for the surgeon and provide the possibility of a symptom-free long life for the patient.

OBSERVATIONS This report discussed two young patients who presented with relatively short-duration reports of ataxia and diplopia. Investigations revealed intraaxial brainstem lesion. During surgery, thick, pus-like fluid was evacuated and part of a wall was resected. Histology revealed that the lesion was an endodermal cyst. Both patients are well and are lesion- and symptom-free for 24 and 72 months.

LESSONS Endodermal cysts are some of the few long-standing and benign intraaxial brainstem lesions.

https://thejns.org/doi/abs/10.3171/CASE2020

KEYWORDS endodermal cyst; brainstem; neurenteric cyst

Endodermal cysts are relatively rare cranial lesions. Their location anterior to the brainstem is more characteristic. Location in the supratentorial compartment is only rarely encountered. In 2005, we reported our experience with seven cases of endodermal cyst, which was the largest experience reported at that time in the literature. ${ }^{1}$ Our current surgical experience with endodermal cyst includes 30 patients treated from 1997 to 2019. Our literature search identified four reports of entirely intraaxial brainstem location. ${ }^{2-5} \mathrm{We}$ report two additional cases of intraaxial brainstem endodermal cysts and discuss their management.

\section{Illustrative Cases}

\section{Case 1}

A 21-year-old man presented with reports of progressively increasing headache, ataxia, and diplopia and inability to move both eyes laterally that had lasted for $\sim 3$ months. When admitted, he was able to walk on his own but was unable to run. Neurological examination revealed spastic quadriparesis. Bilateral sixth nerve paresis was also present. Investigations revealed a large lesion that extended across the width of the pons. The lesion projected and had ballooned into the floor of the fourth ventricle. A small part of the cyst appeared to surface on the anterior part of the brainstem.
The lesion was hypointense on T1-weighted images with some areas of hyperintensity. The lesion was hyperintense on T2weighted images. There was no contrast enhancement (Fig. 1), and restricted diffusion was seen on diffusion images. The patient received surgery by a midline suboccipital approach. The bulging floor of the fourth ventricle was visualized after retracting the tonsils. An incision was made in the thinnest part of the floor, safeguarding the cranial nerve nuclei and brainstem fiber tracts. On incising the cyst wall, greenish pus-like fluid typical of an endodermal cyst flowed out. The entire cyst content was evacuated. A large part of thin capsule was excised. The whole pons appeared to have been split by the lesion. Histological examination revealed an endodermal cyst. The patient rapidly recovered from all his symptoms. At follow-up of 24 months, the patient is well and back to his routine life. Delayed postoperative imaging showed excision of the endodermal cyst (Fig. 1).

\section{Case 2}

A 14-year-old girl presented with reports of headache and progressive left hemiparesis for $\sim 2$ months. She had numbness over the right half of her face and diplopia on looking toward the right for $\sim 15$ days. Neurological examination revealed right-sided V1, V2, and 

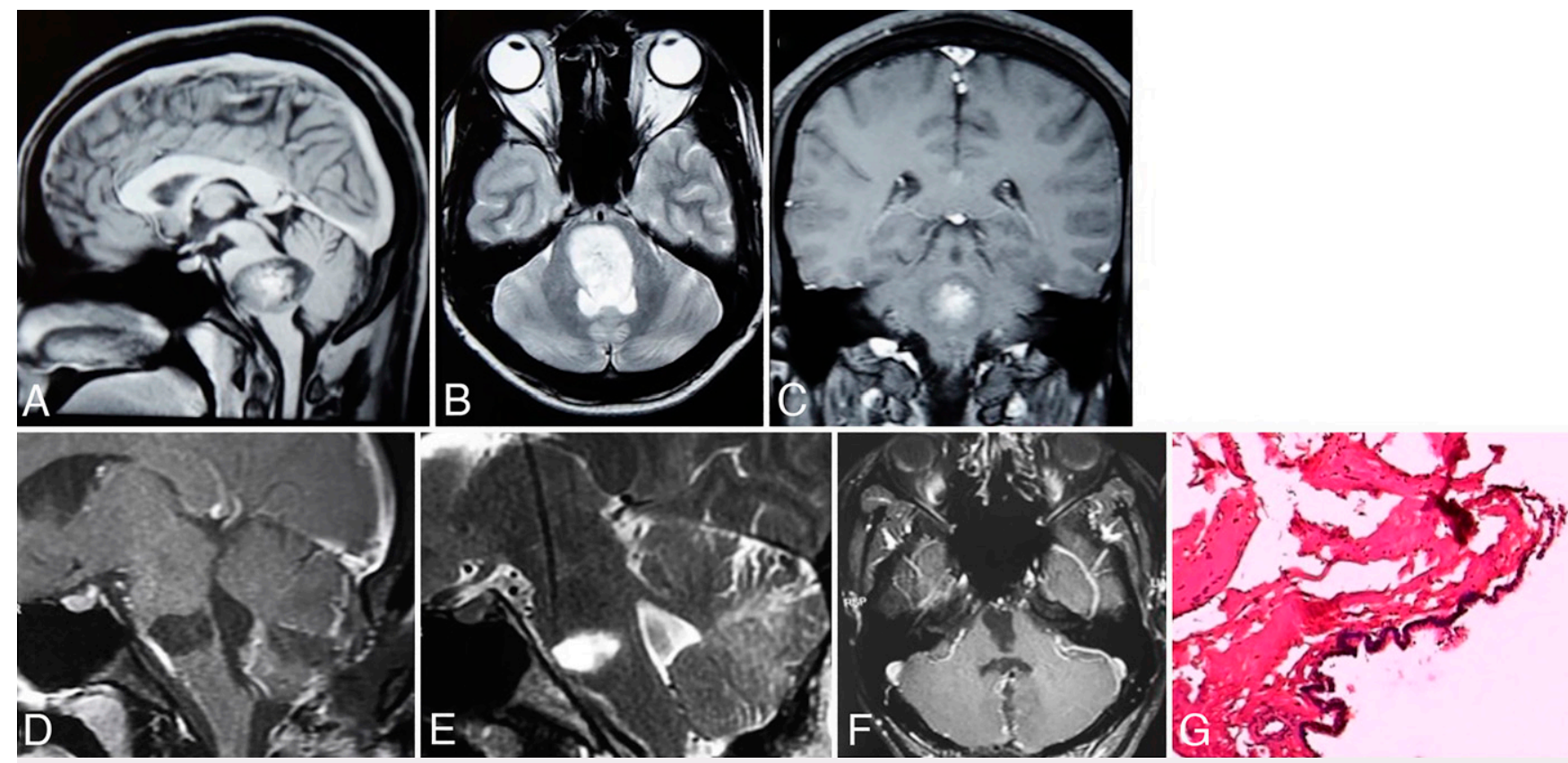

FIG. 1. Images of a 21-year-old male patient. A: T1-weighted sagittal MRI showing the hypointense lesion with areas of hyperintensity in the pons. A small connection can be seen extending from the ventral aspect of the pons to the clivus. B: T2-weighted axial image showing the hyperintense cystic lesion. C: Contrast-enhanced coronal image showing ill-defined enhancement. D: Postoperative sagittal contrast-enhanced image showing excision of the lesion. E: Postoperative T2-weighted image. F: Postoperative axial MRI showing excision of the endodermal cyst. G: Original magnification $\times 400$. Hematoxylin and eosin stain showing endodermal cyst wall lined by pseudostratified ciliated columnar epithelium.

V3 hypoesthesia and right sixth nerve paresis. There was left-sided grade $3 / 5$ spastic hemiparesis. Investigations showed a large cystic lesion in the right half of the pons (Fig. 2). Imaging characteristics were similar to that of Case 1. The surgical route and strategy were similar to Case 1. The cyst content was also similar. Only a small part of the capsule was removed. Histology revealed that the lesion was an endodermal cyst. At follow-up of 72 months, the patient is well and carrying out her routine life. Imaging showed no recurrence of cyst.

\section{Discussion}

\section{Observations}

Endodermal cysts are relatively rare intracranial tumors. ${ }^{1,6}$ Various names, including epithelial, enterogenous, neurenteric, bronchogenic, respiratory, and foregut cysts, have been associated with these cysts. ${ }^{1,7}$ Endodermal inclusion cysts such as Rathke's cleft cyst, colloid cysts, and enterogenous cysts have a common histogenesis but are named differently according to their respective location. Although the cysts simulate neurenteric cysts, which are more often located anterior to the spinal cord, direct evidence of connection with the gastric system is only rarely reported. Several theories have been proposed to explain possible pathogenesis. ${ }^{1-7}$ As with Rathke's cleft cyst, error in embryogenesis appears to be a likely cause for these developmental cysts. Location anterior or anterolateral to the brainstem is more often identified. The possible reason for such preferred location is unclear.

Unique radiological features characterize endodermal cysts. ${ }^{1-7}$ Hyperintensity on T1-weighted images and hypointensity on T2-weighted images are frequently identified. On MRI, the features mimic those of resolving hematoma or an abscess. Soft calcification inside and outside the confines of the cyst is a frequent observation. As a general rule, endodermal cysts do not show restricted diffusion, thus differentiating them from epidermoid cysts. However, diffusion restriction has been infrequently reported in endodermal cyts, as was seen in both of our patients. ${ }^{8}$

Color and consistency of the fluid content of endodermal cysts mimic that of pus in an abscess. The content may sometimes simulate that of liquid or thick inspissated or dried-up pus or partially organized hematoma. Considering the fluid- or semisolid gel-like consistency, evacuation of the cyst content is relatively easy despite its formidable location anterior to the brainstem and in the vicinity of critical cranial nerves and blood vessels. In our earlier article on the subject, we stated that resection of the wall of the endodermal cyst is important but indicated that complete resection may not be pursued if dissection impacted by intimate relationships and inadequate exposure makes this procedure risky. ${ }^{1,6}$

\section{Lessons}

Location within the confines of the brainstem is extremely rare. We could locate only four reports of such intraaxial brainstem location of endodermal cyst. ${ }^{2-5}$ In both of our cases, the preoperative impression regarding the nature of the lesion was that of a pilocytic astrocytoma or even a hematoma, probably related to a cavernoma. Intraoperative identification of thick, pus-like material raised the suspicion of an endodermal cyst. The cyst wall could not be dissected safely off the brainstem in both cases, and only a part was taken for histological examination. Histological examination of the cyst wall confirmed that the lesion was an endodermal cyst.

On retrospective review of the radiological images in both cases, we observed that the anterior wall of the cyst reached the surface 

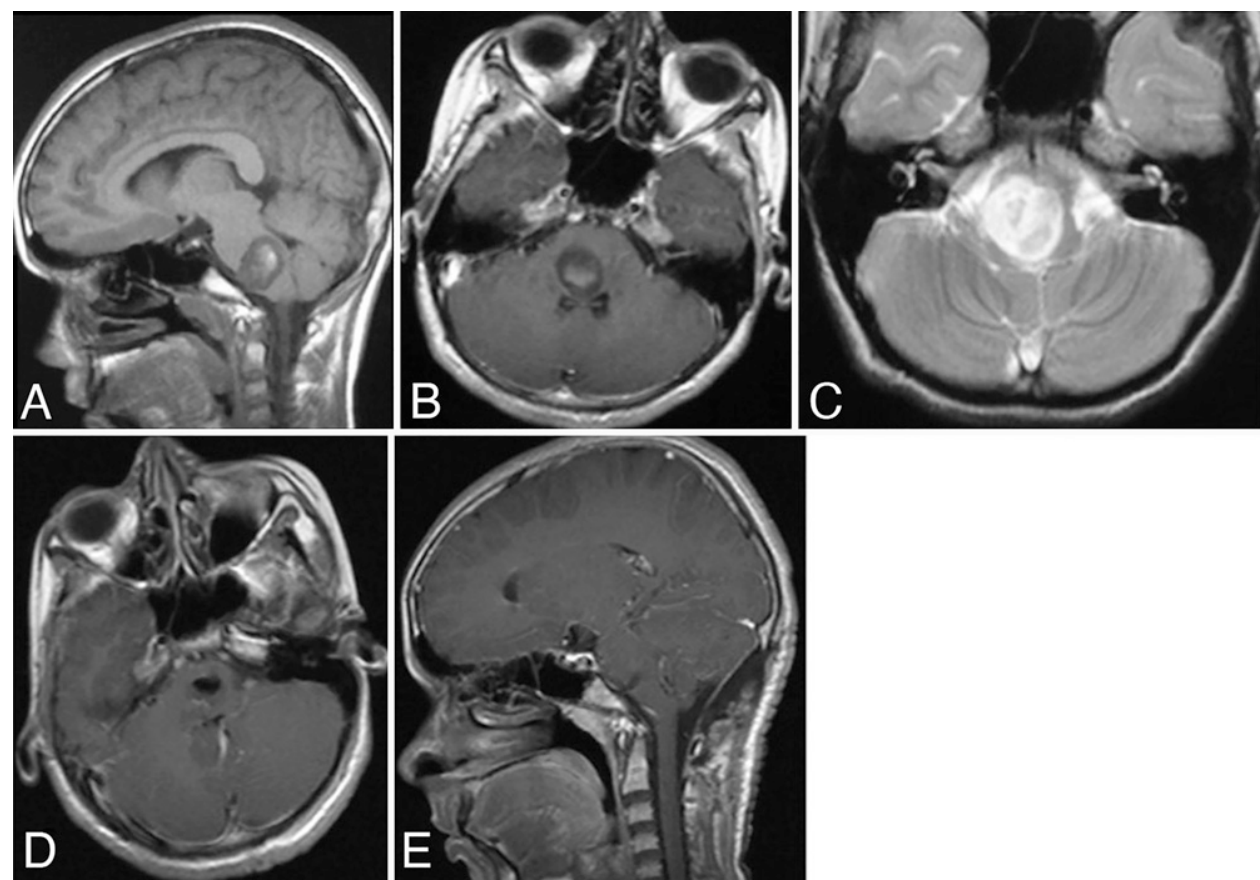

FIG. 2. Images of a 14-year-old female patient. A: T1-weighted sagittal image showing the hypointense pontine lesion with central hyperintensity extending up to the ventral border of the pons. B: T1-weighted contrastenhanced axial image showing no contrast enhancement. C: T2-weighted axial image showing the hyperintense lesion. D: Postoperative contrast-enhanced axial image showing resection of the tumor. E: Postoperative sagittal image showing resection of the endodermal cyst.

of the brainstem and was exposed to or in proximity to the clivus. Considering that the cyst wall was exposed over the anterior surface of the brainstem and that the sequence of growth pattern was apparently from anterior to posterior, one wonders if the incision over the posterior surface of the brainstem could have been avoided and an approach that accessed the anterior surface of the brainstem may have been preferred.

We identified that the recurrence rate of endodermal cysts after evacuation of the entire cyst contents and even partial resection of the cyst wall is low. ${ }^{1}$ In both of our cases, in which follow-up is more than 2 years, there has been no recurrence of cyst or symptoms.

\section{References}

1. Goel A, Muzumdar D, Chagla A. Endodermal cyst anterior and anterolateral to the brainstem: a report of an experience with seven cases. Br J Neurosurg. 2005;19(2):163-166.

2. Agresta G, Sokol D, Kaliaperumal C, Kandasamy J, Gallo P. A novel management proposal for intrinsic brainstem neurenteric cysts: case report. J Neurosurg Pediatr. Published online October 18, 2019. doi:https://doi.org/10.3171/2019.8.PEDS19336.

3. Lach B, Russell N, Atack D, Benoit B. Intraparenchymal epithelial (enterogenous) cyst of the medulla oblongata. Can J Neurol Sci. 1989;16(2):206-210.

4. Wong ST, Moes GS, Yam KY, et al. Intrinsic brainstem neurenteric cyst with extensive squamous metaplasia in a child. J Neurosurg Imaging Tech. 2016;1:26-37.

5. Cho JM, Ahn JY, Kim SH, Lee KS, Chang JH. An endodermal cyst mimicking an intra-axial tumor in the medulla oblongata. Childs Nerv Syst. 2010;26(6):853-856.
6. Shah A, Muzumdar D, Goel N, Goel A. Fulminant fatal meningitis following partial resection of an endodermal cyst. Acta Neurochir (Wien). 2010;152(4):729-730.

7. Bejjani GK, Wright DC, Schessel D, Sekhar LN. Endodermal cysts of the posterior fossa. Report of three cases and review of the literature. J Neurosurg. 1998;89(2):326-335.

8. Hingwala DR, Radhakrishnan N, Kesavadas C, Thomas B, Kapilamoorthy TR, Radhakrishnan VV. Neuroenteric cysts of the brain-comprehensive magnetic resonance imaging. Indian J Radiol Imaging. 2013;23(2):155-163.

\section{Disclosures}

The authors report no conflict of interest concerning the materials or methods used in this study or the findings specified in this paper.

\section{Author Contributions}

Conception and design: A Goel, Shah. Acquisition of data: A Goel, Jadhav, Shah. Analysis and interpretation of data: A Goel, Shah, Shenoy, N Goel. Drafting the article: A Goel, Shah, Shenoy, N Goel. Critically revising the article: A Goel, Shah, Shenoy, N Goel. Reviewed submitted version of manuscript: A Goel, Shah, Shenoy, N Goel. Approved the final version of the manuscript on behalf of all authors: A Goel. Administrative/technical/material support: A Goel. Study supervision: A Goel.

\section{Correspondence}

Atul Goel: K.E.M. Hospital and Seth G.S. Medical College, Parel, Mumbai, India. atulgoel62@hotmail.com. 